\title{
UNIVERSITYOF
}

FORWARD

THINKING

WESTMINSTER用

WestminsterResearch

http://www.westminster.ac.uk/westminsterresearch

"Nanostandardization" in action: implementing standardization

processes in a multidisciplinary nanoparticle-based research and development project

Roubert F., Beuzelin-Ollivier Marie-G., Hofmann-Amtenbrink M., Hofmann H. and Hool A.

This is an author's accepted manuscript of an article published in NanoEthics, 10 (1) 4162, 2016.

The final publication is available at Springer via:

https://dx.doi.org/10.1007/s11569-015-0248-8

The WestminsterResearch online digital archive at the University of Westminster aims to make the research output of the University available to a wider audience. Copyright and Moral Rights remain with the authors and/or copyright owners.

Whilst further distribution of specific materials from within this archive is forbidden, you may freely distribute the URL of WestminsterResearch: ((http://westminsterresearch.wmin.ac.uk/)).

In case of abuse or copyright appearing without permission e-mail repository@westminster.ac.uk 


\section{"Nanostandardization" in action: implementing standardization processes in a multidisciplinary nanoparticle-based research and development project}

\section{Abstract}

Nanomaterials have attracted much interest in the medical field and related applications as their distinct properties in the nano-range enable new and improved diagnosis and therapies. Owing to these properties and their potential interactions with the human body and the environment, the impact of nanomaterials on humans and their potential toxicity have been regarded a very significant issue. Consequently, nanomaterials are the subject of a wide range of cutting-edge research efforts in the medical and related fields to thoroughly probe their potential beneficial utilizations and their more negative effects. We posit that the lack of standardization in the field is a serious shortcoming as it has led to the establishment of methods and results that do not ensure sufficient consistency and thus in our view can possibly result in research outputs that are not as robust as they should be. The main aim of this article is to present how NanoDiaRA, a large FP7 European multidisciplinary project that seeks to investigate and develop nanotechnology-based diagnostic systems, has developed and implemented robust, standardized methods to support research practices involving the engineering and manipulation of nanomaterials. First, to contextualize this research, an overview of the measures defined by different regulatory bodies concerning nano-safety is presented. Although these authorities have been very active in the past several years, many questions remain unanswered in our view. Second, a number of national and international projects that attempted to ensure more reliable exchanges of methods and results are discussed. However, the frequent lack of publication of procedures and protocols in research can often be a hindrance for sharing those good practices. Subsequently, the efforts made through NanoDiaRA to introduce standardized methods and techniques to support the development and utilization of nanomaterials are discussed in depth. A series of semi-structured interviews were conducted with the partners of this project, and the interviews were analyzed thematically to highlight the determined efforts of the researchers to standardize their methods. 
Finally, some recommendations are made towards the setting up of well-defined methods to support the high-quality work of collaborative nanoparticle-based research and development projects and to enhance standardization processes.

\section{Keywords}

Nanoparticle-based research and development, Collaboration, Standardization methods, Standard operating procedures, Regulation, SPIONs

\section{Introduction}

People are exposed on a daily basis to nanoparticles from a wide range of sources such as diesel exhaust particulates, and tobacco smoke. In the view of the European Commission which have attempted to provide a general definition, nanomaterials, refer to materials in which more than $50 \%$ of the particles by number distribution have at least one external dimension smaller than $100 \mathrm{~nm}$, while nanoparticles have three external dimensions smaller than $100 \mathrm{~nm}$ [1]. Nanomaterials, and more specifically soft nanoparticles and nanostructures, have been under research and development (R\&D) for approximately 40 years [2]. Nano-sized materials have attracted much interest in the medical application field because their physical, chemical, and biological properties may significantly change in the nano-range, thereby enabling new or improved diagnoses and therapies. Most of the nanostructures used for therapeutic applications (i.e. nanopharmaceuticals) that have been available to the general public for around 15 years use conventional substances that have already been approved but can be more effectively formulated and are more soluble and less toxic when used as part of a nanoformulation. Nowadays, nanopharmaceuticals are becoming increasingly complex and can be customized for particular organs or cell types by using targeting molecules in combination with the receptor of the cell surface [3]. They can penetrate a cell by carrying bioactive molecules or by crossing natural biological barriers such as the blood-brain barrier [4]. 
Nanoparticles may also be used for a range of diagnostic applications [5]. Inorganic nanoparticles may show characteristics such as quantum effects that change, for instance, the specific wavelength of emitted light or the magnetic behavior, as in iron-oxide nanoparticles, which become superparamagnetic at sizes below $15 \mathrm{~nm}$ [6]. Superparamagnetic iron-oxide nanoparticles (SPIONs) can act as a contrast agent for MRI owing to their superparamagnetic properties and, thus, can be used for detecting various diseases [7]. It is this property that NanoDiaRA, a large FP7 European multidisciplinary project aimed at investigating and developing nanotechnology-based diagnostic systems, is seeking to exploit. The R\&D processes of NanoDiaRA are the subject of the present paper.

However, nano-sized materials, nanoparticles or more complex nanostructures such as core/shell particles functionalized with biomolecules, pose major safety challenges to human health and the environment. In the European community, the European Medicines Agency (EMA) published in June 2006 a seminal reflection paper on nanotechnology [8]. Since 2006, the European Food Safety Authority (EFSA) has been also closely following developments in nanotechnologies and shared its scientific position in March 2009 [9]. It suggests that nanomaterials should be assessed for risk, considering exposure, at five different levels as their state may evolve in varying conditions. The Food and Drug Administration (FDA) acknowledges that if in principle nanoscale materials present regulatory challenges similar to those of products using other emerging technologies, these challenges may be magnified in the case of nanomaterials because their properties might change continuously as the size enters the nanoscale range [10].

Thus, the burning issue for nanomaterials is to determine whether they should be treated as any other new material or whether the challenges they pose in relation to their size require specific measures and to establish what these measures should be. Although a number of research publications have examined methods to define the potential risks of nanomaterials, the detection and evaluation of these risks and the regulatory measures to be implemented [11], there still exists an information deficit, particularly concerning nanoparticle characterization methods. "Nanotechnology entails new 
toxicological risks which are vaguely defined and difficult to test, a field in which our knowledge about immune defense response - if it is able to react at all in any given situation - is poor" [12]. This is particularly critical for complex nanostructures for which understanding the biodistribution, biopersistence, and clearance behaviors is essential. Hence these complex nanostructures raise key challenges with regards to the definition of appropriate methods and standards for their characterization and preparation [13].

Engineered nanoparticles raise particular concerns of their own as they could be highly reactive and mobile within the human body [14]. Although it is well known that engineered nanoparticles can be taken up by different types of cells and could lead to toxic reactions, the evidence is disparate and the mechanisms of uptake are still only partially examined. We posit that, although there has been considerable progress in this area in the past few years, there is still a shortfall in terms of research concerning the development of reliable, reproducible and standardized methods and models with regards to the production, characterization and testing of engineered materials. This article aims to address these issues by presenting valuable insights in a particular project that uses engineered ironoxide particles as contrasting agent.

Our aim in this paper is to present the efforts of our collaborative European FP7 multidisciplinary research and development project in the area of nanotechnology-driven diagnostics to rise to the challenges with regards to the standardization of methods to create, define, characterize, test, and manipulate engineered nanoparticles. NanoDiaRA aims to develop a novel technology to detect the molecular processes that may cause the inception and progress of arthritis. This technology is based on the synthesis and use of functionalized SPIONs to image the inflammatory events of arthritis and thus identify biomarkers associated with the joint disorder. The SPIONs are produced at Ecole Polytechnique Fédérale de Lausanne (EPFL) and distributed to fifteen different partner locations around Europe (see Figure 1) for conducting experimental work to investigate the imaging properties and, thus, seek to develop the technology in a pre-competitive stage. One of the major challenges for 
the Powder Technology Laboratory (LTP) at the EPFL, where the nanoparticles are produced, is to obtain an all-encompassing Good Laboratory Practice (GLP) accreditation, which is very challenging because of its status as a university laboratory accessible by a wide range of academics. If the LTP does meet GLP regulations concerning the traceability of materials, additional standardization measures have been enforced in this laboratory and the other sites involved in this project to ensure that the particles are engineered and manipulated at the highest level. Thus, this work presents the overall efforts made in this project to standardize the various experimental practices involved in the engineering and manipulation of SPIONs to provide the highest possible standards.

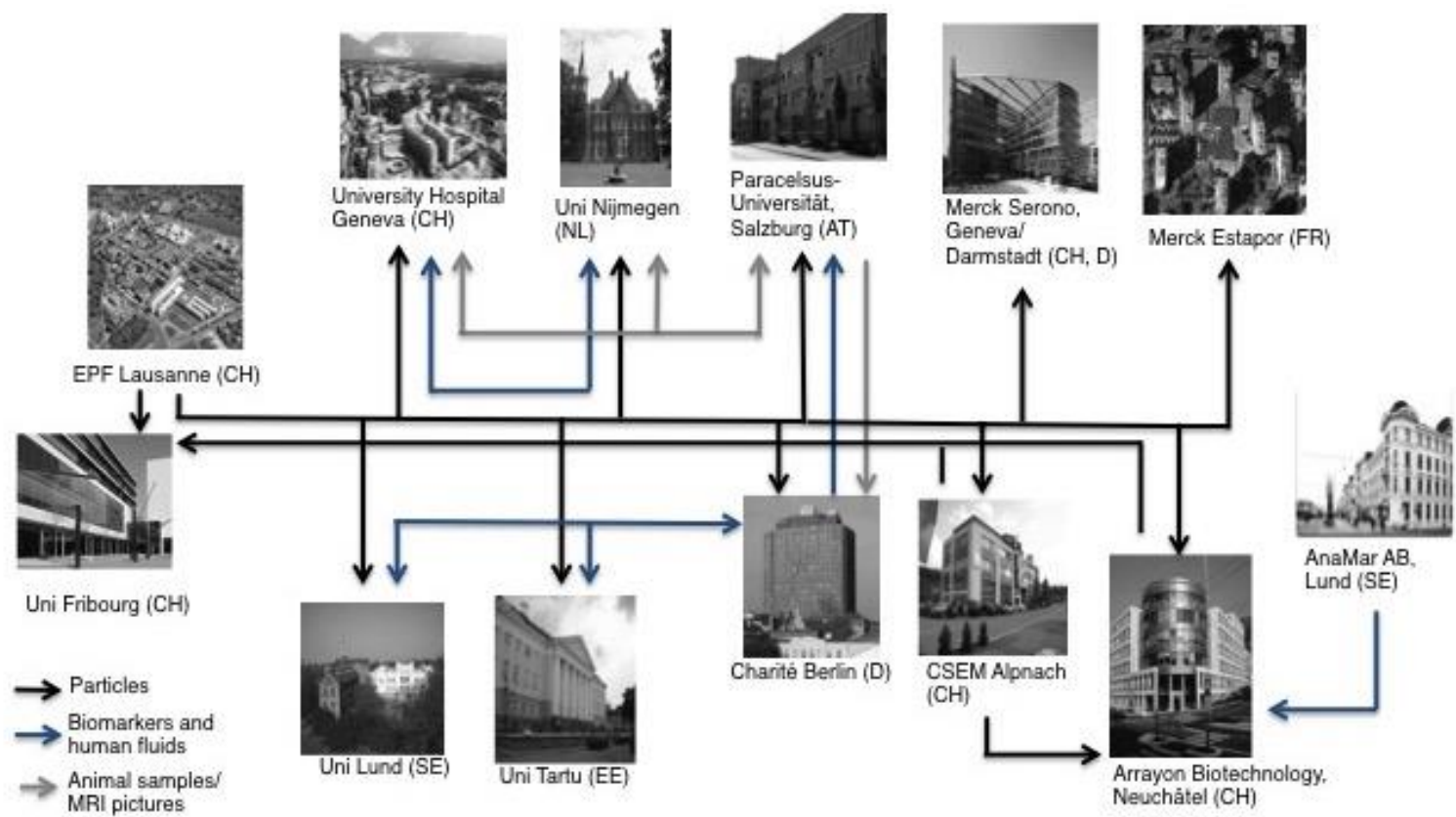

Figure 1: Excerpt of the sample flow in NanoDiaRA. SPIONs are sent from EPFL to different partner groups; biofluids and animals may be treated with nanoparticles when sent away for other tests.

First, an overview of the regulatory frameworks in Switzerland and abroad is presented to examine the various rules, directives, and guidelines that have become effective in the last several years and steer the development and manipulation of nanomaterials for research projects in the nanomedical field, like NanoDiaRA, the project under consideration here. Second, a number of prominent research projects that probe the use of nanoparticles for medical applications and that have adopted interesting 
standardization processes at various levels, are subsequently reviewed to identify examples of good practice in which standardization has played an essential role. Third, this paper focuses on the efforts made by NanoDiaRA to standardize the various experimental practices involved in the production and handling of engineered nanoparticles, the characterization methods used to accurately determine the properties of the engineered particles and the early toxicity tests introduced to guarantee essential safety. The positive effects on the project resulting from the introduction of these standardization efforts are also explored. Finally, a series of guidelines and recommendations is presented with a view to inform practitioners on how standardization in this field can be adopted in practice to improve consistency in the scientific work undertaken.

\section{Regulatory framework for nanomaterials}

In recent years, several national and international regulatory bodies and related special initiatives have attempted to provide a regulatory framework to enable the handling of nanomaterials in a standardized manner. This section presents a brief selection of existing regulatory measures concerning the definition, production, characterization, and use of early-development-stage nanomaterials with safety being a major concern. The section draws from a range of relevant examples from various stages of the research and development of these materials; these examples are relevant to NanoDiaRA and similar projects at different levels such as the international, European, and national levels. This section does not pretend to be exhaustive but rather aims to present the regulatory settings which can be applicable to European projects which probe the use of nanomaterials for medical purposes such as NanoDiaRA. Consequently, it focuses on the international and the European contexts but also on the Swiss level as, apart from being an important site of activity for NanoDiaRA, it provides some interesting insights and elements of discussion which could be of interest to practitioners in the field. 


\subsection{Global standards}

Standardized testing, measuring, and assessment methods are necessary to describe, measure, and predict the effects and complex behaviors of nanomaterials in the human body, in organisms, and in the environment. As this paper is concerned with standardization issues in the field of nanomaterials, the focus is first directed to the guidelines provided by the most influential bodies, such as the Organisation for Economic Co-Operation and Development (OECD) and the International Organization for Standardization (ISO), which regulate the development of testing and measuring techniques to evaluate the impact of nanomaterials on human health and the environment.

The OECD has stipulated a set of useful international testing strategies and guidelines with a concern for the impact of nanomaterials. Particularly, the object of the OECD Working Party of Manufactured Nanomaterials (WPMN), established in 2007, is to develop methods and strategies aimed at contributing to the safety of nanomaterials for people and the environment. They also aim to consider emerging issues that are relevant to policies in science, technology, and innovation related to the responsible development and use of nanotechnology, which they define themselves as structures and systems of size "typically less than 100 nanometres" [15]. The OECD program has heavily focused on generating appropriate methods and strategies to reduce potential safety issues. These include the establishment of an OECD database on manufactured nanomaterials, on the testing of specific nanomaterials in relation to human health and safety, and on the setting up of appropriate in vitro and in vivo testing methods [16]. A thorough review has been conducted to ascertain whether the test guidelines developed to determine the physico-chemical, toxicological, and ecotoxicological properties as well as the environmental behavior of chemicals are equally applicable to nanomaterials [17]. In this preliminary review document, the properties of 13 frequently used nanomaterials are analyzed within a so-called sponsorship program. The analysis includes chemical/physical properties, acute and chronic toxicity and ecotoxicity endpoints, and environmental behavior properties. The analysis results are used to produce recommendations for the amendment of existing test methods and 
the development of new, standardized test methods. The OECD primarily focuses on nanomaterials that have already been developed or are commercially available. However, by creating guidelines for the standardized testing of these materials, interesting methods emerge as consequences. For example, the OECD recommends 200 defined "endpoints" in in vivo studies and provides detailed documents for "Testing and Assessment" strategies [18].

The norms and quality standards proposed by the ISO are present in everyday life and affect a broad range of domains. As a global organization focusing on standardization, the ISO performs important and recognized groundwork in the field of nanotechnology. Since 2005, it has been running a technical committee for nanotechnologies aimed at ensuring greater standardization in this field. It includes the "understanding and control of matters and processes at the nanoscale, and the utilization of the properties of nanoscale materials that differ from the properties of individual atoms, molecules, and bulk matter, to create improved materials, devices, and systems that exploit these new properties" [19]. In particular, the committee has developed and published standards for terminology and nomenclature in nanotechnology [20]. Their remit also covers metrology and instrumentation, including specifications for reference materials; testing methodologies; modeling and simulations; and sciencebased health, safety, and environmental practices. For instance, methods for particle-size measurements including sampling rules and definitions of corresponding terms are specified in a special annex [20]. The ISO classifies materials at the nanoscale, which they define as sizes between 1 and $100 \mathrm{~nm}$ similarly to the definitions of REACH and OECD, by using a classification system that considers the dimension, structure, and chemical and physical properties [20]. In the development process, nanomaterials for use in pharmaceutical products have to comply with specific rigorous criteria that are stricter than the criteria for other materials used in the industry. The International Conference on the Harmonisation of Technical Requirements for Registration of Pharmaceuticals for Human Use (ICH) defines the tests substances have to pass to be used in medical applications for humans [21]. 
It is to be noted that ISO standards mainly emphasize on industrial application rather than on basic research and therefore it assumes that much more developed processes are in place. In addition, ISO standards do not reflect the actual material and laboratory requirements which need to be implemented for the research to be conducted. The pragmatic approach that we are putting forward is thought to provide a valuable complement to the standards imposed by official bodies and thus to lead to more practical and applicable guidelines for the field of basic research that probes the use of nanomaterials for medical applications.

Another widespread safety measure promoted both at the international and national levels, e.g., in Canada, Germany, UK, Switzerland and US, for the manipulation of nanomaterials is the use of safety data sheets (SDS/MSDS). Their aim is to ensure that individuals who handle or work with chemical substances comply with safety measures regarding health protection. It is governed by the Globally Harmonized System (GHS) which defines and classifies the hazards of chemical products, and communicates health and safety information on labels and safety data sheets. In the EU, the European Chemicals Agency (ECHA) makes thorough recommendations on how SDSs should be written and recorded [22], while in Switzerland, this is ensured by the FOPH or FOH [23]. In December 2010, a first version of nano-SDS recommendations taking into consideration the latest knowledge in the nano-field was published and an update of the document was released in April 2012 [24].

In summary, the guidelines and recommendations provided by international bodies, such as OECD, ISO, and ICH, or global safety measures such as the use of SDSs are essential in nanomaterial research as they provide high-level orientation with regards to safety in relation to the definition, production, characterization, and utilization of nanomaterials The next step is to explore the regulatory provisions made at the European level. 


\subsection{Working at the European level}

NanoDiaRA brings together the efforts of a number of sites distributed across Europe which exchange nanomaterials to conduct their experimental work. Thus the European regulatory system, which has much to contribute in this field, is considered next, and examples are discussed to provide relevant European regulatory background to this work, as it is directly relevant to the work that NanoDiaRA is conducting, as well as more generally to the large number of European research efforts that also investigate the use of nanomaterials in the medical field.

As previously mentioned, the European Commission has attempted to clarify the terminology of nanomaterials and published in 2011 a recommendation for the term "nanomaterial" [1], the implications of which have since been discussed widely on national and European platforms [25]; [26]; [27]; [28]. One particularly interesting European initiative in the form of a large-scale integrating European FP7 project, NANODEFINE, is currently seeking specifically to develop a standardized approach to support the implementation of the EC's recommendation for the definition of nanomaterials. It aims to develop robust and reliable standardized methods and instruments to measure the size of particles in the range of $1-100 \mathrm{~nm}$ with diverse shapes and coatings for a wide variety of materials in different media and products [29].

In the EU, nanomaterials are governed by a number of ordinances and directives. Some of them already contain nano-specific provisions, while amendments are still being discussed for others. Sectoral legislations as well as different regulation "cultures" make the regulation of nanomaterials a very multifaceted topic even within the EU [30]. Moreover, nanomaterials are also regulated "vertically," i.e., specific to the product group they are aimed to be produced for, such as cosmetics and food [31].

Nanopharmaceuticals in Europe are subject to national pharmaceutical regulation as well as to the EU Pharmaceutical Legislation, as coordinated by the EMA mentioned earlier. In addition, the European 
Regulation on Registration, Evaluation, Authorisation and Restriction of Chemicals (REACH)

addresses the production and use of chemical substances in general and their potential impacts on both human health and the environment. To date, it is the strictest law regulating chemical substances, and it will affect industries throughout the world. REACH was established in June 2007 with a phased implementation planned over the subsequent decade. It is estimated that characterization data are lacking for $86 \%$ of the chemicals, and the REACH process aims to address this fundamental issue.

The European Commission appointed a panel of experts in 2009, whose duties were to examine whether the test data and methods currently required by REACH are relevant for the evaluation of the risks associated with nanomaterials. The RIP-oN projects [32]; [33]; [34] are independent projects funded by the Institute for Health and Consumer Protection (IHCP) of the European Commission's Directorate General Joint Research Centre (JRC). They declared that it is not their aim to establish definitions concerning nanomaterials and that they are themselves anticipating a common European terminology. For their studies, however, they consider particles and tubes with at least one dimension $<100 \mathrm{~nm}$ as nanomaterials [32].

The two projects on "Specific Advice on Fulfilling Information Requirements for Nanomaterials under REACH" [33] and "Specific Advice on Exposure Assessment and Hazard/Risk Characterisation for Nanomaterials under REACH" [34] already show where the emphasis is placed: ensuring transparency and access to open information and proper risk evaluation. With regards to the broad testing of new and existing materials, "integrated test strategies" are recommended [33]. However, these strategies are aimed at the different effects of nanomaterials on animals, humans, and the environment. They are developed, for instance, to evaluate acute toxicity, dermal adsorption, or eye irritation, but they do not include characterization at a basic level [35]. In addition, REACH focuses on materials produced in large amounts, and therefore, the attempts for a comprehensive testing scheme do not apply to the small quantities produced at an early research level to be upscaled only in a later production stage. 
Other European initiatives, like the ongoing large-scale integrating FP7 project, NANOREG, specifically seeks to answer the concerns of regulators and legislators regarding the Environmental Health and Safety issues related to nanomaterials. The project aims to develop a set of reliable instruments specifically designed for the risk assessment, characterization, toxicity testing, and exposure measurements of nanomaterials and to develop long-term testing strategies in collaboration with authorities and the industry [36]. The efforts made by the NANOREG have been extended through the ongoing Horizon 2020 initiative PROSAFE, which aims to provide additional support for risk assessment, management, and governance in the area of nano-safety. It endeavors to encourage the acceptance of Safe By Design all over the EC and to carefully consider all regulatory developments that emanate from the safety challenges posed by the convergence of nanotechnologies and biotechnologies [37].

If the EU provides a regulatory background in the nanotechnological research area that informs the practitioners and can be relevant in specific cases, the national regulatory context defined by Switzerland also ought to be explored, as the site at which the nanoparticles used within the entire NanoDiaRA are produced is located in Lausanne, Switzerland. In addition, this national context is deemed worthy of consideration here as it provides interesting insights on regulatory directives put in place in a particular country to provide additional safety measures for the use of nanomaterials

\subsection{Operating in Switzerland}

The Ecole Polytechnique Fédérale de Lausanne, EPFL, where the nanoparticles for NanoDiaRA are produced, is a leading Swiss institution for the safety of nanomaterials and.thus, the handling of particles at the production site is regulated by Swiss Federal law. A number of measures put in place by the Swiss Federal Government are discussed here with the aim to inform the research community of the legislative systems that provides the local regulatory context for the NanoDiaRA project and to give examples of good practice. 
According to the Swiss Federal Office for Public Health (Bundesamt für Gesundheit, BAG), synthetic nanomaterials come under existing legislations concerning their area of use, such as legislations on chemicals, foodstuff legislations, environmental legislations, and legislations on therapeutic products. These legislations regulate the common aspects of the respective fields, such as usage restrictions, emission limits, and foodstuff expiry. However, this can be slightly problematic as the particles produced in R\&D projects such as NanoDiaRA are at an early stage of development, the application for their future use is not always fully defined and they can be used for a range of purposes. For instance, superparamagnetic nanoparticles can be used for different types of biomedical applications, such as contrast agents and tools for targeted drug delivery, and also as instruments that can destroy unhealthy tissues such as cancerous tumors in the body through heating.

In the framework of an "Action plan for synthetic nanomaterials," the Swiss Federal Offices consider the amendments necessary to detect nano-specific problem areas that require further regulatory provisions [38]. The action plan was developed by the Federal Office of Public Health (FOPH), the Federal Office for the Environment (FOEN), and the State Secretariat for Economic Affairs (SECO) in cooperation with an inter-departmental task force and the involvement of a panel of scientific and economic experts. It was adopted by the Federal Council in 2008, and in 2012, it was extended until 2015 [39]. The objectives of the action plan include the development of a regulatory framework for the handling of synthetic nanomaterials, creation of scientific and methodical specifications aimed at identifying and preventing potential harmful effects on health and the environment, promotion of a public dialogue on the opportunities and risks of nanotechnology, and better utilization of existing tools for the development and implementation of sustainable nanotechnology applications. Different projects have been implemented within the scope of the action plan, covering safety, precaution, and disposal topics. Consequently, Switzerland is now perceived as one of the leading countries in the regulation and standardization of nanomaterials and this justifies why we consider it to be worthy of discussion in this paper as it can provide some fascinating insights. 
. For instance, the FOPH and the FOEN created a working group consisting of several experts from different Swiss institutions that are leaders in the research and regulation of nanomaterials, such as the Swiss Institutes of Technology in Lausanne and Zurich (EPFL and ETH), the Swiss Federal Laboratories for Materials Science and Technology (Empa), and the Swiss Agency for Therapeutic Products (swissmedic).

A precautionary matrix was designed to help evaluate the nano-specific health and environmental risks of nanoproducts. This matrix has been devised to help the industry, regulating themselves in relation to their employees, consumers, and environment, by enabling the structured assessment of the "nanospecific need for precautions" when handling synthetic nanomaterials. Interested parties can answer specific questions about the handled materials concerning their properties, life cycle, exposition of workers, and consumers. The precautionary matrix is based on modules that include a number of evaluation parameters, whereby the potential effect of a nanomaterial is estimated using its reactivity and stability. The probability and degree of exposure of human beings and input into the environment are examined through data on the physical surroundings of the particles, the amount marketed, and the emission of particles during production or use [40]. The matrix is regularly revised and updated based on experiences and new scientific knowledge [41]. Based on this matrix, EPFL has developed a detailed regulation for researchers and workers on how to handle engineered nanoparticles [42]; [43].

In summary, Swiss national attempts to regulate the handling of nanomaterials focus primarily on risk assessment, environmental safety, and safe working conditions. However, this should not be the only concern. Reliable and standardized testing is also of great value for the overall scientific community if conducted within a solid international normative context that reduces the differences between local variations, as it will be discussed in great detail in relation to NanoDiaRA under consideration here.

This brief high-level review of the regulatory field appears to indicate that if different national and international instances have introduced some interesting regulatory provisions for the definition, production, characterization, and use of nanomaterials, they cannot always be applied directly when 14 
working on exploratory research that involves the early development of nanoparticles, because they may not be of the right scope. However, it has to be acknowledged that they offer useful directions and provide guidelines, which can be adapted for use at a project level, on top of standardization measures, which ought to be locally adopted. The next section provides a selective review of some of these measures as introduced in specific projects.

\section{Previous efforts for nanomaterial-based development}

As discussed previously, guidelines and recommendations from official bodies in the nanomaterial field constantly evolve. The establishment of such standards is also one of the objectives of several national, European, and international projects dealing with nanoparticles. Some establish guidelines and others present overviews of various studies regarding the toxicology of nanomaterials. The outputs of workshops that specifically focus on these issues, such as [44] and [45], should also be considered as they can result in valuable contributions.

An EU project and two national projects, which are of particular relevance, will be presented as three case studies in the following sections. These are the FP7 project NANOMMUNE, which produced a quality handbook for the Standard Operating Procedures (SOPs) for nanoparticles [46]; the British Defra project REFNANO, which created a list of essential reference materials [47]; and the GermanSwiss project DaNa, which established quality criteria [48]. Also worth mentioning are the FP7 projects NANOVALID [49], MARINA [50], and ITS NANO [51] for their efforts in promoting the use of higher standards. The integrating project NANOREG [36] and its Horizon 2020 extension [52] is also acknowledged for promoting standardized risk assessment, management and governance in nano-safety as previously mentioned. Many other projects also establish SOPs and other standardized practices in the course of their research; however, many of these standardization efforts have not been reported and, thus, are not accessible for further use. In most of these national and European projects, the time for compiling and disseminating project-related information and knowledge is restricted because of tight time constraints. These restrictions result in many preliminary findings on 
standardized processes and methods not being published in peer-reviewed journals, and the internal data might be only used if one or more partners continue in the field. In addition, the widespread use of time-limited contracts with collaborators hinders the continuity.

This section aims at reviewing the efforts of some of these research projects in contributing to the definition and implementation of standard methods and procedures to develop, handle, and test nanoparticles. It is by no means an exhaustive review; rather, it seeks to shed light on examples of good practice.

\subsection{Standard operation procedures: the basis for further standardization?}

Standardized protocols are recognized as indispensable by many researchers involved in projects related to nanomaterials [48]. Even though the past few years have seen a rising number of projects in the nanomaterials field that have published standardized protocols - in part due to increased scrutiny and pressure from funding institutions - there still appears to be a deficit in information concerning the standardization of procedures in this domain of enquiry.

For conventional chemical products, a wide range of experimental protocols are available, particularly concerning the testing of their toxicity, which is of paramount importance. However, these protocols are not directly useable in the field of nanomaterials owing to the specificities of nanoparticles [53]; [54]; [45]; [44]. Regardless of whether existing protocols for substances other than nanoparticles can be modified and applied to nanomaterials, whether they should just be used as a source of inspiration, or whether new protocols need to be designed from scratch, Warheit et al. put forward a very helpful and functional approach when dealing with the testing of nanoparticle-based materials [55]. In these scheme a series of questions be asked:

1. Which components need to be tested, i.e., the particle, coating of the particle, or both together? 
2. Which types of tests need to be carried out, i.e., in vitro tests, in vivo tests, or a combination?

3. Should surface modification be included?

4. Should particle dissolution be used?

Noticeably, as part of testing work in the FP7 project NANOMMUNE, Harald Krug and his team released an entire "Quality Handbook" with "Standard Procedures for Nanoparticle Testing" [46], which collects all the experimental protocols for the synthesis and evaluation of the nanomaterials used in this project into a full portfolio of protocols including synthesis, characterization, and in vitro, in vivo, and in silico testing. All of them contain a "safety procedure and precautions" part, which quickly describes specific recommendations to follow while performing the experiment, as well as a references section. Synthesis SOPs describe a full step-by-step protocol.

A consensus seems to have been reached on the suitability of the REACH guidelines provided by the EU (see section 2.2) for the development of SOPs, as many project consortia advocate the compliance with these guidelines to produce high-quality, reliable, and reproducible standardized procedures [48]; [54].

Another interesting approach is followed by the US National Cancer Institute (NCI). On its Web portal [56], which aims at fostering the sharing of information among nanotechnology researchers, over 40 protocols have been made available for assays in the field of nanoparticles aimed for clinical application. To manage the process of developing and maintaining protocols in a collaborative manner, a wiki was designed and implemented. Protocols are divided into eight categories: in vitro assay, in vivo assay, physico-chemical characterization, radiolabeling, safety, sample preparation, synthesis, and others. Guidelines are provided on how to search for a protocol, create a new one, or modify an existing one. It is possible to not only update a protocol but also protect it by operating a "visibility option" which will make it private and only modifiable by its author. 
The project DaNa is an example of a shared project between several institutions from Germany and Switzerland [57] ${ }^{1}$ which illustrates the implementation of very valuable initiatives with regards to the evaluating of SOPs. By using a testing platform, standards and protocols were tested and settled up to improve the collection of data regarding nanomaterial toxicology. A "Methodology of selection, acquisition and evaluation of publications in project $\mathrm{DaNa}$ " was put in place to collect relevant analyses data [48]. This guideline for publication includes mandatory assessment criteria (e.g., rawmaterial CAS number and specific information regarding the tested material), as well as desirable ones, such as the declaration of used SOPs. The data obtained can then be compared and fully understood, and proper conclusions can be drawn.

These examples provide different approaches for designing well-defined standardized operating protocols to develop and manipulate nanoparticles and avoid potential risks. They all highlight the importance of providing the scientists producing these protocols with consistent guidelines on how safety can be ensured. Characterization is another essential area where very valuable attempts have been made at introducing a certain level of standardization, as discussed next.

\subsection{Characterization and reference materials}

As soon as the agreed protocols have been set up, the material under investigation needs to be defined by its properties, characteristics, and reactivity with the environment. The establishment of reference materials is crucial to enable comparability, reproducibility, and repeatability (see also section 4.4.1). According to the definition given in the amendment to ISO Guide 30, a reference material is a

\footnotetext{
${ }^{1}$ Partners of project DaNa are DECHEMA e.V. Frankfurt/Main, Fraunhofer Institute for Ceramic Technologies and Systems Dresden, Helmholtz Center for Environmental Research Leipzig, Karlsruhe Institute for Technology EggensteinLeopoldshafen, Independent Institute for Environmental Concerns Berlin (Germany), and Empa St. Gallen (Switzerland).
} 
"material, sufficiently homogeneous and stable with respect to one or more specified properties, which has been established to be fit for its intended use in a measurement process" [58].

The British Department for Environment, Food and Rural Affairs (Defra) established a list of reference materials as part of the project REFNANO that can be used for calibration, metrology, and method validation and development [47]. As another major source, the German Federal Institute for Materials Research and Testing (BAM) is, in cooperation with external partners, feeding the results of its work and that of third parties into a database for "Nanoscaled Reference Materials," which, amongst others, comprise DIN and ISO standards [59]. Reference materials should be established in parallel while developing the characterization methods and used as standardized control mechanisms.

When a reference material is available, nanoparticles can be characterized using customary standard routines. The definition of certain properties is the basis of characterization, and these tend to be determined for all nanomaterials. They are referred to here as "primary properties" and include the size, shape, charge composition, purity, and stability. The methods for measurements, however, depend on the complexity of the formulation of the material and need to be adapted depending on the material and its composition. Interferences with the test systems, especially biological interactions, can occur and produce misleading results, e.g., in cell-based assays [54]. For example, the pre-testing of endotoxin content using at least two standardized methods at an early stage of nanomaterial development could avoid unnecessary experiments and allow decisions regarding the material's acceptance for in vivo testing. Proper reproducibility of the primary properties of each nanomaterial both physico-chemical and biological - is of high relevance, as even small deviations of these properties may ultimately have a large impact on the in vivo effect. The particle's in vivo stability and the drug release rate are of particular relevance.

The characterization of nanoparticles involves specific challenges and risks, as the unique properties of particles can result in unwanted behaviors such as agglomeration and loss of stability. While "standard" characterization describes the nanoparticles and defines their properties, toxicology defines 19 
their in vivo behavior; thus, it characterizes the material from a biological viewpoint. Compared to characterization, toxicology is more focused on the effect of nanomaterials on the environment, microorganisms (ecotoxicology), animals, and humans. The starting point is often determining which unit should be used, e.g., amount of particles per volume and mass of particles [34]. When testing in vitro, the biokinetic behavior of the material also plays an important role. Additionally, it is not always clear which amount of particles actually interact with the tested cells, because, for instance, particles can float on the cell culture, and therefore, some particles do not even touch the cells [60].

While primary properties are common to all nanomaterials, others are specific to the target. For instance, each organ has its own characteristics and may react differently to different nanoparticles, i.e., certain characteristics of particular nanoparticles may have a larger influence on some organs than on others [61]. For instance, the fiber length and rigidity of carbon nanotubes do not allow them to pass through stomata [62]. Thus, varying measurements may be needed depending on the objectives.

Furthermore, a widely accepted classification of nanomaterials would be useful to establish adequate reference materials for different purposes. The NCI lab recommends that all aspects of newly characterized nanomaterials should be studied to allow for the establishment of ranges of admission for the produced batches [53]. These recommendations emphasize that each nanomaterial should be considered as something completely new, and all analyses must be adapted on a case-by-case basis. The idea of the NCI lab was to fill in the gaps and educate the research community on the thorough characterization of nanomaterials. This approach contrasts with the one of most industrial bodies such as the Nanotechnology Industries Association (NIA) for US and Canada which tend to produce rankings of nanomaterial with a view to standardize the analysis by using read-across and analogue data to increase transparency, consistency and alignment [63]. It is also to be noted that classifications in industries are often linked to their future applications. The complex field of toxicology can only be supported by the careful characterization of nanomaterials, a thorough understanding of the possible mechanisms, and the planning of each experiment to the very last detail. Common mistakes can be 
avoided by carefully planning the analysis and considering all parameters that may influence the results of each test [53].

\subsection{Need for an integrated approach}

The examples considered here suggest that nanotechnology in our view still actively necessitates regulatory measures, greater control, and standardization. Much of the work done thus far focuses on one or two aspects; there are only very few comprehensive studies in this area at this point that embrace all the facets of nanotechnology, including regulation, methods, definition, characterization, toxicity, safety, and product information. One notable exception is NANOREG and its extension PROSAFE, which both seek to investigate these issues extensively and provide adequate support to regulators, legislators, and the scientific community. However, at the project level, comprehensive reports summarizing the methodologies and methods undertaken in the project that do not simply describe research results are frequently missing. This is often caused by the somehow hurried completion of the project and the lack of funds or motivation to publish the knowledge acquired.

Suitable testing methodologies are, for a large part, developed in parallel and in close collaboration with research practitioners. The presented studies emanated from projects in different locations that were eager to share knowledge. We posit that all the aspects of nanotechnology that have been covered need to be integrated in a more comprehensive approach to obtain a more complete picture of the field of study, particularly in a consortium where interactions and exchanges between members are the basis for productive work. Having reviewed previous attempts at defining clear standards for the development and characterization of nanomaterials and having highlighted examples of good practice in the last sections, in the following, an in-depth analysis of NanoDiaRA is conducted as a particular European FP7-funded project, which has placed standardization at the heart of its activities.

\section{An integrated standardization approach in the NanoDiaRA project}


This section explores how the NanoDiaRA FP7 project has set up and implemented standardization processes to enable various partners to synchronize their working practices and conduct harmonized research activities that lead to the generation of more reliable data and production of results of higher quality.

NanoDiaRA is a large multidisciplinary project that aims at developing nanotechnology-based diagnostic tools to support the detection and treatment of rheumatoid arthritis and osteoarthritis. As described earlier, the SPIONs used across the consortium are synthesized and characterized at the EPFL in Lausanne and sent to several other partners for various purposes. They are shipped to the University of Geneva (Switzerland), where they are used in animal studies, to a company in Neuchâtel (Switzerland), where tests for the development of an array are conducted, to the Charite University in Berlin (Germany) for in vitro testing, to the University of Lund (Sweden) for biomarker studies, and finally to the University of Nijmegen (Netherlands) for the development of a mouse model.

From the inception of the project, strict guidelines for "improving and monitoring research exchange/ethical issues" were established across the different partner sites involved in the project [64]. Precise guidelines on how to record and document the experimental work in a standardized format across the consortium were also introduced from the beginning, with a strong emphasis on good scientific practice and on the continuous maintenance of standardized laboratory books across multiple sites. In addition, the work conducted at the EPFL was used as the basis to develop the first prototype of an electronic sample book (ESB), i.e., an electronic IT system used to centralize and disseminate recommendations on how to send materials to and from various sites and how to handle the particles upon receipt. Every new batch of particles produced at the EPFL site would be thoroughly recorded in the ESB alongside supporting data such as the quantity of particles, their properties, and a range of acceptance (RoA) that defines boundary values for the usage of the nanomaterials [65]. This would allow not only for each sample sent to be monitored carefully but also for the high standards of handling of the nanoparticles implemented at EPFL to be disseminated to other partners. 
It is essential to note that the national and international regulatory measures and standards such as ICH, REACH, OECD, and ISO - explored earlier providing rigorous guidelines to handle nanomaterials safely and to conduct high-quality testing - are used in NanoDiaRA as an optimal threshold, rather than literally. Indeed, NanoDiaRA is an exploratory project in which it is not realistic to expect strict standards often used in industrial production to be applied at each level of R\&D. For instance, in the NanoDiaRA project, the LTP aims at implementing good laboratory practice (GLP) as minimum standards. However, an all-encompassing accreditation is difficult to attain for an academic laboratory that operates as part of a university. One of the requirements would be to separate the labs used for educational purposes from those used for research, development and production, which are not possible in an academic institution where the laboratories are accessible by a range of members of the academic staff. Yet, the LTP does meet GLP rules related to traceability - i.e., the recording of used chemicals, lot numbers, etc. - and places much of emphasis on ensuring the reproducibility of the conducted studies by setting up internal guidelines ensuring appropriate standards. This tension between complying with international standards, which are not always applicable in the practice of an exploratory project such as NanoDiaRA, and the handling of local constraints and contingencies that emerge from this type of project justify why is it essential for such a project to introduce and implement local standards and measures in order to ensure a consistently high quality of the produced research outputs in a safe environment.

In order to examine in great detail the experimental procedures in operation in this project and the efforts to introduce standardization to support these practices consistently, a number of consortium members were interviewed. Some of these interviews were conducted in the laboratories as scientists were performing their experimental work while others were small group discussions. All of them were recorded, transcribed, and qualitatively analyzed using thematic analysis [66]. The findings of this investigation point at three main areas: the setting up of unified standardized protocols called SOPs and their continuous evaluation; the use of a reference batch of the synthesized nanomaterial to define an RoA for the properties of every subsequent batch of particles; and finally, the meticulous 
conducting of preliminary biological toxicity tests and the systematic production of certificates of analysis (CofA).

\subsection{Experimental processes: protocols and standard operating procedures}

The experimental protocol is an essential artifact used by scientists to design and conduct experimental activities. It provides a platform for the researcher to keep a record of the experimental setup used, the equipment required, the various materials necessary to conduct the experiments, and the different tasks to be performed. It is often a dynamic document that is frequently revised as the experimental work evolves. The level of standardization between various protocols used within a project can significantly vary. The NanoDiaRA project, developing a new contrast agent as a diagnostic tool, has put effort into keeping protocols consistent among different research teams. The utilization of SOPs in situ to support the experimental work in NanoDiaRA is described in this section to shed light on the practices involved in their creation, editing, and utilization in this project and to evaluate the impact of the standardization on the quality of the experimental work.

\subsubsection{Initialization of SOPS}

The setting up of every new experiment was observed to start with the development of a draft experimental protocol to outline briefly the operations necessary to conduct the investigatory work. "You sort of have a rough idea of how you want to set up your experiment, generally from something you have read in an article. So you start by writing down a few ideas" (Scientist A). One of the main drivers for designing a new SOP is quite often the specialized literature and the experimental methods and techniques described in relevant articles. "You keep on reading papers which are relevant in your field and from there you often pick up a few ideas for your own experiments" (Scientist B). Thus, the procedures presented in the various scientific publications are seen as trustworthy processes because they have been approved by the scientific community and hence can be used as a reliable basis for setting up one's own experimental work. However, an adaptation process is often necessary to tailor 
the experimental activities to the local conditions and the experimenter's needs. "You get the main idea from what you read but then you have to adapt it to what is available in your lab, to the equipment that you have there. Sometimes they suggest you should use that material but you don't have it, so you try with something else" (Scientist C).

\subsubsection{Coordinated protocol development}

We observed that, in certain cases, the experimental work to be undertaken could be closely related to earlier ones conducted by other members of the consortium or those being conducted simultaneously in another lab. The scientist usually contacts the lab of the involved partners to know more about their work and about the availability of the research protocols used to undertake the experimental work. In this case, a multidirectional communication is set up towards the design of a particular experiment and the dynamic and iterative creation of the supporting SOP. The experimenter typically uses the partners' initial ideas, basic description of methods, or fully designed protocols to set up their own experiment, test their hypothesis, and author a new SOP. As the experiment is in progress, it is customary for scientists to contact their partners to enquire about particular techniques, solve specific problems, or ask for clarifications. "You try your own thing based on what they have sent you but so often you have to ask them for help. Normally I send them an e-mail to ask them about a problem. Sometimes, if I can use the phone in the lab, I call them" (Scientist B).

As the project evolved, a more formal process of generating, storing, and sharing protocols was introduced. The scientists across projects were required to follow a series of guidelines to produce consistent SOPs, which would then be stored centrally in an electronic format in the ESB for general access. This sample book was introduced in the project to standardize the authoring of protocols and to improve the sharing of procedural resources to coordinate the experimental work better. Tighter validation processes were also introduced [67]. 


\subsection{Electronic management of SOPs}

Once a reasonably stable version of the SOP is in place, it is used to support the overall experimental process. Experimenters use it as the basis to prepare the experiment at the bench, i.e., to locate the right equipment and the right material. The eventual differences between materials prescribed in the SOP and those effectively available in the lab and used in the experimental work are meticulously noted in the scientist's lab book. The SOP is modified at a later stage, making it a continuously evolving document with different versions. The ability to store documents in an electronic format in the ESB allows SOPs to be retrieved and modified when required and for new versions to be managed collaboratively. This provides the experimenters with the flexibility to use a trial-and-error approach for their experimental work set-up, to keep a record of all their attempts, to share them with others, and to manage the various produced versions of the SOPs.

\subsection{Validation of protocols}

Validation plays an essential role in experimental design and, thus, in the development of supporting protocols. It is embedded at different stages of the collective experimental setup with different levels of formalization. The testing for experimental validity is systematically conducted and organized around two concepts - repeatability and reproducibility - as summarized in Table 1. As a starting point, repeatability is the ability of a scientist to conduct a given experiment repeatedly and to obtain similar results each time, while reproducibility is the capacity for an experiment to be carried out again by a different scientist or team in a different environment with a different setup and to achieve analogous results.

\begin{tabular}{|l|c|}
\hline Local Repeatability & - Same lab \& same scientist \\
& - Same material \& same apparatus \\
\hline Local Reproducibility & - Same lab \& different scientist \\
\hline
\end{tabular}




\begin{tabular}{|c|c|}
\hline & - $\quad$ Same material \& same apparatus \\
\hline Distributed Repeatability & $\begin{array}{l}\text { - } \quad \text { Different lab \& same scientist } \\
\text { - } \quad \text { Same material \& different apparatus }\end{array}$ \\
\hline Distributed Reproducibility & $\begin{array}{l}\text { - } \quad \text { Different lab \& different scientist } \\
\text { - } \quad \text { Same material \& different apparatus }\end{array}$ \\
\hline
\end{tabular}

Table 1: Experimental validation in distributed projects.

\subsubsection{Local repeatability and reproducibility}

During experiments, scientists thoroughly document the ongoing process in their lab book by taking note of sample quantities, equipment calibration levels, early observations, and initial results. All these elements are then used to set up and refine the draft SOP. Once this initial SOP is established, the scientist typically repeats the experiment several times in the same lab conditions to evaluate whether the results can be replicated and improved. "Once you have your initial protocol, you tend to do the experiment again and again to see if you can get the same results and the adjustments you have to make to get better results" (Scientist D). Thus, the scientist engages in a typical experimental cycle based on trial and error to evaluate and improve the quality of their procedure, which is the foundation of the iterative development of experimental SOPs.

Similarly, the scientists frequently consult their colleagues and ask them to follow the protocol and undertake the procedures in the same lab conditions and using the same equipment. The aim is to ensure the reproducibility of the experiment and, thus, the quality of the written SOP based on an identical experimental setup but undertaken by a different person. Initially, the systematic level of this validation process varied from one scientist to another. "Once you have an experiment that you are pretty confident with and that seems to produce similar results every time you do it, you ask one of your colleagues if he can have a go and if he can follow the protocol. That helps you assess whether 
your experiment is on the right track" (Scientist E). With time, as the NanoDiaRA project advanced and communication between the various partners became more frequent and natural, these validating processes became further embedded within the project, and various experimenters adopted them in an increasingly systematic manner.

\subsubsection{Distributed repeatability and reproducibility}

In the case where a scientist works closely with partners and there is interdependence between their experiments, it is quite standard for the initiating scientist to visit their partner and attempt to recreate the experiment in their local setup. "As part of a work package there can be an experiment which I need to do first and then send them my results because they need them to do their own work. Then we can organize for me to go there to visit them in their labs. I try to repeat the experiment I have done but in their labs with their equipment to show them but also to see if it works" (Scientist D). Beyond the demonstration purpose, such distributed repeatability is a way for the experimenter to evaluate the quality of their SOP and to test their experimental design in different settings and potentially adjust it to refine the results. Again, exchanges of these types became more standardized as the project unfolded to allow experimenters to share their practices with partners involved in the collective experimental setup. In other situations, similar experiments have to be conducted in various labs that take part in the project. In some instances, these experiments are very similar, while in others, they differ to some extent. These situations offer the opportunity for the validation of experimental protocols by ensuring distributed reproducibility. "We work closely with this other lab as part of this work package. We do our experiments and they do very similar ones over there. We came up with the initial SOP. Then we sent it to them and asked them to try it on. We asked them to tell us whether it made sense to them and whether they got similar results and if not to tell us about the differences" (Scientist A). Hence, either the initial SOP can be modified or a new one can be created to refer to the previous one. This can create a network of interconnected SOPs that have been thoroughly tested and 
can be archived. We observed, yet again, that as the project gained momentum, these validation processes became increasingly systematic.

\subsection{Quality of engineered nanomaterials: biological characterization and early toxicity tests}

Beyond the implementation of tighter processes concerning the design, validation, and use of procedures, a wide range of additional measures were introduced with regards to the synthesis of nanomaterials at one site to be used by researchers at other sites to ensure the quality of the produced materials.

\subsubsection{Reference batch and range of acceptance $(R o A)$}

Because of the nature of the experimental setup in this project, standardized systematic processes had to be implemented. "We produce the particles that the other members of the consortium are going to use for their experiments. So we need to make sure that we follow proper procedures so that we produce materials of consistently high quality" (Scientist A). First, an SOP is produced and refined after several attempts to produce a master batch of nanoparticles that complies with precise requirements specified as part of the project. This is aimed at being used as a reference for further batches developed subsequently. "You always keep a sample of your master batch and this is your reference for any future work" (Scientist A). This reference batch of particles is fully characterized to establish a very precise understanding of the synthesis and accurately define their sizes and charge. The produced particles are physically and chemically characterized immediately after the synthesis of the core particles. A process of coating particles may occur much later, and the coated particles are also characterized.

A certain number of initial batches are then produced, characterized, and compared to the initial reference batch. This leads to the definition of a RoA, which can then be used as a standard against which the subsequent batches of synthesized particles can be measured. "Every time you produce a new batch you look in which range your new batch is. If it's out of this range, then you don't accept 
$i t$ " (Scientist D). These RoA values are recorded alongside the results of the characterizations for every batch of new particles. Thus, the experimenters who will use them can precisely determine how the particles behave compared to the standard batch. For every new batch, both the actual result of the batch characterization and the RoA are stored in the ESB platform to allow access to any project member. This is used for further reference and, thus, contributes to enhanced traceability.

It is, however, possible for the properties of a batch of particles to vary with time. This is the reason why multiple characterizations of a batch are carried out at various time points. "We distinguish the characterization of the initial production from later characterizations because our particles may evolve and it is important for us to keep a track of that" (Scientist B). The initial characterization is thus guaranteed for a period of time, considering that the particles are stored following specific conditions mentioned in the SOP. New characterizations need to be undertaken after that period of time to determine whether the particles have evolved, and the RoA can thus help establish whether the quality has been compromised.

\subsubsection{Preliminary toxicity tests (PTTs)}

If a batch of nanoparticles is to be sent to members of the project consortium who intend to use them as part of in vivo experiments, it is essential that the particles are thoroughly tested for toxicity. A series of preliminary toxicity tests (PTTs), which are conducted at the production site before sending out the particles, have been introduced at different time points to guarantee safety. As mentioned in section 3.2, early quality testing can avoid unnecessary investigations. PTTs consist of cell viability tests. "You cultivate cells, and when they have grown, you add the nanoparticles to your cells. You leave them for a certain time for interactions and then, after a certain time of incubation, you carry out the test and see how the cells react" (Scientist A). Reference tests with the same type of cells and a master batch of particles are used as a standard against which subsequent tests can be compared. "Because all the previous tests were done with the HeLa cells, we always keep the HeLa cells and our master batch as the control" (Scientist B). 
Subsequent PTTs may be undertaken on different instances once the particles have been generated. It is essential for the tests to be conducted under conditions that are close to the ones under which the particles are to be utilized. "If the experiment that is going to be carried out is in blood, then you need to test your particles in blood to try to have a medium as similar as possible to theirs" (Scientist E). Thus, relatively complex initial testing can lead to an early decision point, which helps determine whether the development of a given material should be continued. If the decision to go ahead is taken, further tests are conducted.

If a new batch is produced for direct experimental use at another site, a PTT is conducted, and the results are carefully recorded before the particles are shipped across. If a batch of particles has been produced earlier and stored for a period of time, a new toxicity test is conducted to guarantee that the particles are stable and have not become toxic. At times, another test is conducted by the partners receiving the particle, if they have the required facilities, to ensure that the toxicity is still at an acceptable level and that the particles are still safe to use. These various iterations of tests are recorded centrally using the ESB to allow the highest possible transparency and traceability.

\subsection{Certificate of analysis (CofA)}

In order to facilitate and standardize the diffusion of the information relevant to batches of a particle, the use of CofA was introduced. A CofA can be defined as a document that verifies the compliance of a product to certain specifications to certify the quality of that product. Thus, every new batch of generated nanoparticles is given a CofA, which summarizes the characteristics of these particles in a visual tabular format. This includes the results of the physico-chemical and colloidal characterization for this particular batch alongside the RoA to show how this particular batch compares to the original one. It can also include the results of the PTT, if the particles are meant to be used in humans or animals. This CofA is physically sent alongside the batch of particles to the receiving laboratories. In addition, an electronic copy of the CofA is also uploaded on the ESB for interested scientists to know more about a particular batch. This allows the logging of any change that may have occurred in the 
state of the particles and hence in their characterization or in their toxicity levels so that the latest information is always available. This strict linkage between the actual material and the supporting information both in physical and digital formats ensures that the overall stock of nanomaterials is accurately and efficiently managed.

The in-depth analysis of the standardized procedures introduced by the NanoDiaRA project to align the different work practices of various members and to enhance the quality of the experimental work can be used in our view as 'lessons learned' and 'examples of goof practice' that ought to be shared and can inspire a series of guidelines and recommendations that may be relevant to the work of other scientists in this field.

\section{Guidelines and recommendations}

\subsection{Design, development, and use of standard operating procedures}

In the light of the discussions on both regulatory measures and previous standardization efforts, it is clear that the Standard Operating Procedures (SOPs) play an essential role in research and development. Ideally, laboratories involved in nanomaterial engineering and research ought to comply with GLP. However, in an academic environment, it is often difficult to fulfill all the conditions as expected in the industry because some rules are simply not applicable, owing to the ways a university research laboratory operates. Still, the fundamental concepts of reproducibility and repeatability can be adapted for the specific needs of R\&D to ensure high quality.

For ease of use, it is preferable that all SOPs used in a project have a similar structure. Furthermore, it is important to adapt the document to the different backgrounds of the individuals involved for a better understanding. The description of the methods used in the protocol should be clear but with sufficient details to enable use by all researchers involved in a project, including those with a slightly different research expertise. A template should be used with comments indicating how to fill the form, the terminology to be used should be made clear, and different conversion units for the presentation of the 
results should be suggested. This is essential because in a project or a lab working on engineered nanomaterials, various research disciplines are often overlapping and intertwined because of the complexity of the field. Therefore, the same result, depending on the way it is presented, can be interpreted in different ways. This is the reason why a clear unit system should be specified.

In order to enhance the quality of the research design and development, a four-step approach to the design of the experimental protocol is recommended. In a first instance, the scientist who sets up a new experimental procedure should directly ensure its local repeatability himself/herself. This suggests that the experimenter should conduct the experiment several times with the same material to verify that the same results are obtained each time. This should then be used to refine the protocol and improve it. Subsequently, another scientist should conduct the experiment in the same lab conditions to test local reproducibility. Similarly, the process of executing the same experimental procedure on numerous occasions by another person should be of great benefit to improve the protocol. This should then be followed, if possible, by the validation of the protocol by the original experimenter in a different lab with different conditions to verify that the protocol shows evidence of distributed repeatability. Finally, the protocol should be shared with other partners at different sites, who should also perform the experiment in their own lab conditions to determine whether it is reproducible in different lab conditions when performed by a different person. This approach would hopefully help sharpen the written SOP and improve the quality of the experimental process.

When synthesizing new nanoparticles, each batch should be produced following a well-defined procedure and properly characterized. A specific reference to the used protocol has to appear in the documentation; a good integration of the documentation should support proper traceability. The setting up of a platform such as the electronic sample book (ESB) in NanoDiaRA can be of great value to allow for the centralization and easy retrieval of all available SOPs.

In the past several decades, a number of projects dealing with engineered nanomaterials have been executed; however, there has been little evidence of the availability of usable elements from these 33 
projects, except from what is presented in their final reports. This means that the knowledge acquired during the years of the project has not always been exploitable, because of varying levels of inaccessibility. It is strongly suggested that a consortium should plan for a period before the end of the project dedicated to the dissemination of acquired knowledge, as these inter-project exchanges are essential in a field such as nanotechnology. The centralized recording of SOPs also shows the willingness for full transparency with respect to all the methods used and how the experiments were performed. The ESB used in NanoDiaRA is only an example of how centralization and exchanges between researchers can be digitally supported, and similar approaches can be followed by other projects in the future. Beyond that, it would be recommended that all SOPs should be made publicly accessible online as a useful complement to all the other project data usually made available. After having published all relevant results in peer-reviewed publications upon the completion of the project, NanoDiaRA has decided to follow this approach and to post easily retrievable versions of their SOPs on the DaNa website (www.nanopartikel.info).

Owing to the context of a challenging field and the continuous development of engineered nanomaterials, the documentation must evolve in close relation with the methods. A versioning and archiving system for SOPs is suggested here as an ideal solution to assist with continuously evolving documentation. In combination with the ESB, this provides traceability of the developed methods in correlation with the batches. While older versions remain accessible for further documentation, the latest version is made the active one, thereby avoiding confusion between the different documents.

\subsection{Development and characterization}

In the synthesis of materials such as nanoparticles, it is considered a good practice to produce a master batch and store it carefully for further reference. A complete physical and chemical characterization of this master batch should be conducted to determine the size and charge of the particles with great precision. It is recommended that a certain number of initial batches be produced subsequently and also fully characterized. This enables the definition of an RoA, which can be used as a reference point 
for every subsequent batch that is produced in order to ensure good quality. It is essential to keep this master batch carefully stored and to characterize it fully at regular intervals to ensure that it is stable and that its properties have not evolved. This should be extended to the batches of particles that are produced and potentially used by other scientists. Furthermore, these should be characterized on a regular basis to verify that their properties have not been modified.

To provide proper characterization of developed particles and enable comparability of particle properties and testing results, it is important to ensure the continuity of these properties. To guarantee this, the material must be checked regularly at several time points. Tests can be quick and simple, but the results should be representative of the final state condition of the material as described, for instance, in the PTT, which should be introduced as in the NanoDiaRA project. PTTs should include a reference to standard materials and appropriate controls to avoid any misleading information. Some tests may be performed at two or more locations but have to remain comparable.

Even small deviations in the production and handling of the materials can lead to major differences in the final particle properties. Therefore, it is essential to conduct initial tests, establish ranges of acceptance, and create CofAs for every new batch of particles. As the properties can change over time, it is also important to repeat some of these tests when a particle is used after a certain storage duration.

A quality check is crucial and necessary before sending any samples to a partner; all results obtained should be compiled in the CofA. Any person receiving a shipment should be able to check the RoA of the product to determine whether the material they received complies with the established standards.

\subsection{Exchange in collaborative projects}

In distributed collaborative projects, it is important to be aware of every relevant step that a scientific enquiry has undertaken and of all the tests that have been conducted. Therefore, it is relevant to trace a particular particle's physical route fully within the project as well as each result that has been obtained and manipulation that has possibly been made on this particle. 
The shipment of nanoparticles may cause modifications to the toxicity levels because of less controlled conditions within which the materials are posted. Even though digital probes can be used to record and monitor the temperature and other conditions, additional safety measures should be taken. It is recommended that tests are performed before shipment and upon receipt to ensure that its toxicity level is still suitable. Similarly, toxicity tests should also be repeated if the sample was stored for a period of time as its stability and, therefore, its toxic properties might have evolved.

In large collaborative projects such as NanoDiaRA, a batch of particles may have a long and complex lifecycle through a project and may have been sent to and from different sites to allow different experiments to be performed. All these complex exchanges and the recording of experimental results should be made transparent and visible when the exchanges are occurring but also in retrospect, when these processes may have been conducted repeatedly and with different batches of particles. Therefore, it is recommended to have a two-fold approach: first, to enclose the necessary documents with a physical sample to give direct information to the person who receives the material. To assist the exchange of samples and provide the right information, standard CofAs should be introduced. Second, there should be a documentation system that records each step and the associated experimental results and ensures full traceability of the particles and other samples.

With the help of an ESB, such as the one used in NanoDiaRA, tests at the receiver's end, as described above, can be integrated in the project and queried in a standardized manner when necessary. While the general condition of the sample, i.e., if it is broken or intact is prompted as a standard question on receipt, other conditions have to be checked depending on the sample type. The sender can fill in the relevant information to appear initially with the sample at the receiver's end, and the feedback in question can directly be solicited and then compared by both parties. In this manner, the ESB functions as a "feedback tool" that ensures direct and well-defined responses from the project partners. Feedback is also crucial for engineered nanomaterial development as different results at the receiver's end can be explained by their characteristics. Thus, the laboratory tasked with the synthesis and 
development of the nanoparticles can adapt and proceed to slight modifications to fulfill the project requirements.

In projects such as NanoDiaRA, which aim to develop particle formulations within the course of the project, it is not clear from the beginning which formulation might be used in the end. This can even change several times during the project, depending on the results that are obtained from the different partners' locations, which might only become usable after some time. Thus, the partner producing the particles should always keep a certain amount of a particle formulation, even if it seems that the initial results are not entirely promising. From this "master batch," new batches can be produced if necessary. This must be reflected in a structure that can be described as a "Master and-Child" setup: samples that are sent to partners are children of the master batches. Master batches can be recharacterized whenever required, and new results or properties changing over time will also be applicable for the samples that are already derived from a master batch and sent to other partners. This means that properties of the sent samples are still required to be updated after they have been sent, and it is even possible that the properties mentioned in the CofA undergo change.

An electronic system should be put in place to support a "Master-and-Child" structure, as it was done in the NanoDiaRA project. The system can be used to store and interconnect all the documents such as CofAs and handling instructions directly with the respective sample. The system is also able to trace a sample's complete route through the project: each partition, transport, and all important results that are obtained. Results and properties that are of high relevance to the other partners can be made visible by directly entering and displaying it with the information on the sample, whereas detailed information can be added by attaching the relevant documents. All methods for obtaining the results can also be added in the form of SOPs or other structured descriptions to ensure full reproducibility of the results.

At many instances, the knowledge and skills required for particular experiments need to be transferred to other partners. The SOP remains an important vehicle in such cases, but the need for an exchange platform is crucial: e-mails or phone calls are not always sufficient to describe properly specific 
handling details of a technique. At times, a personal visit and the organizing of training is necessary to ensure proper technology transfer, that the method is reproducible in another environment and when performed by another scientist, and the necessary adjustments are made. There is a double benefit to this approach. First, it helps enhance the quality of the designed experimental protocol through the validation of distributed reproducibility, as illustrated earlier. Second, these direct interactions between partners working on a project from different angles and viewpoints help each party to get a better understanding of the work of their collaborators and thus foster solid cooperation links from which the project as a whole can directly benefit.

\section{Conclusion}

Guidelines have been derived from the study of the standardization practices in NanoDiaRA with a view to enlighten scientists and research practitioners involved in projects or any other research initiatives that may face similar issues. Table 2 summarizes our findings and recommendations towards the increased standardization of methods and processes in nanomaterial research.

\begin{tabular}{|l|l|l|}
\hline Areas & Summary of guidelines & Additional resources \\
\hline $\begin{array}{l}\text { Design, development, } \\
\text { and use of SOPs }\end{array}$ & $\begin{array}{l}\text { Define a consistent structure and layout, } \\
\text { possibly with the use of a template }\end{array}$ & $\begin{array}{l}\text { Templates can be found in [48] }{ }^{2} \\
\text { and [36] }\end{array}$ \\
\cline { 2 - 3 } & $\begin{array}{l}\text { Use terminology and unit system } \\
\text { understandable by everyone }\end{array}$ & E.g. in [68], [69], [20] \\
\hline
\end{tabular}

${ }^{2}$ The SOPs for NanoDiaRA will be available on the DaNa website in early 2016. 


\begin{tabular}{|c|c|c|}
\hline & $\begin{array}{l}\text { Ensure local and distributed } \\
\text { repeatability and reproducibility }\end{array}$ & $\begin{array}{l}\text { Suggested procedure in section } \\
4.3 \text { of this paper }\end{array}$ \\
\hline & $\begin{array}{l}\text { Centralize and share SOPs, possibly } \\
\text { through an electronic platform, to allow } \\
\text { open access and collaborative authoring }\end{array}$ & $\begin{array}{l}\text { Electronic Sample Book (ESB) as } \\
\text { implemented in NanoDiaRA; see } \\
\text { [65] }\end{array}$ \\
\hline & $\begin{array}{l}\text { Set up a well-defined version- } \\
\text { management system to support } \\
\text { collaborative authoring }\end{array}$ & $\begin{array}{l}\text { Versioning system developed and } \\
\text { implemented as part of } \\
\text { NanoDiaRA; see [65] }\end{array}$ \\
\hline \multirow[t]{5}{*}{$\begin{array}{l}\text { Development and } \\
\text { characterization }\end{array}$} & $\begin{array}{l}\text { Produce a reference batch and provide } \\
\text { batch characterization }\end{array}$ & [70] \\
\hline & $\begin{array}{l}\text { Frequently test reference batch to ensure } \\
\text { that characterization remains stable, and } \\
\text { update properties, if applicable }\end{array}$ & [70] \\
\hline & $\begin{array}{l}\text { Conduct early preliminary toxicity tests } \\
\text { and decide early if more tests are } \\
\text { required }\end{array}$ & [71] \\
\hline & $\begin{array}{l}\text { Define a range of acceptance to be used } \\
\text { as a reference for every subsequently } \\
\text { produced batch following the reference } \\
\text { batch }\end{array}$ & $\begin{array}{l}\text { Suggested approach in section } 4.4 \\
\text { of this paper. Further regulatory } \\
\text { steps are under discussion, see } \\
\text { e.g. [72] }\end{array}$ \\
\hline & $\begin{array}{l}\text { Produce a certificate of analysis with } \\
\text { details on toxicity levels }\end{array}$ & $\begin{array}{l}\text { Suggested approach in section } 4.5 \\
\text { of this paper. Further regulatory }\end{array}$ \\
\hline
\end{tabular}




\begin{tabular}{|c|c|c|}
\hline & & $\begin{array}{l}\text { steps are under discussion, see } \\
\text { e.g. [72] }\end{array}$ \\
\hline \multirow[t]{5}{*}{$\begin{array}{l}\text { Exchange in } \\
\text { collaborative projects }\end{array}$} & $\begin{array}{l}\text { Conduct toxicity tests before sending } \\
\text { and after receiving particles }\end{array}$ & Examples in [73] \\
\hline & $\begin{array}{l}\text { Send the certificate of analysis and } \\
\text { range of acceptance alongside the batch } \\
\text { of particles physically as well as } \\
\text { electronically }\end{array}$ & $\begin{array}{l}\text { Monitored by use of ESB; see } \\
{[65]}\end{array}$ \\
\hline & $\begin{array}{l}\text { Set up a feedback to inform about the } \\
\text { quality of the batch and compliance with } \\
\text { their requirements. }\end{array}$ & Supported by ESB; see [65] \\
\hline & $\begin{array}{l}\text { Set up a sample-exchange-management } \\
\text { system, possibly through an electronic } \\
\text { platform, to track and monitor the } \\
\text { sending and receipt of batches }\end{array}$ & Supported by ESB; see [65] \\
\hline & $\begin{array}{l}\text { Provide interdisciplinary training for the } \\
\text { scientists working in collaborative } \\
\text { projects }\end{array}$ & $\begin{array}{l}\text { Organization of summer and } \\
\text { winter school as for NanoDiaRA; } \\
\text { see [74] }\end{array}$ \\
\hline
\end{tabular}

Table 2: Summary of recommendations for the standardization of methods and processes in nanoparticle-based research.

Our key recommendations thus emphasize on three areas: 
(1) the design and development of standardized SOPs which should be consistent, unambiguous, accessible by all and enforce repeatability and reproducibility both locally and globally;

(2) the methodical development batches of particles which should be interconnected through a clear scheme and characterized and tested at regular intervals to monitor their potential change in states;

(3) the exchange of materials which should be centralised and thoroughly monitored potentially through the use of an electronic platform which can efficientlly keep a track of all physical and digital artifacts within the project.

This pragmatic approach as put forward in this paper which is very much practice-driven aims to provide a useful complement to the official guidelines provided by existing standardization organizations. The intention is to offer practitioners in the field of nanomaterial research for medical applications practical recommendations that they can adopt in their daily scientific practice.

\section{Acknowledgements}

The research leading to these results has received funding from the European Community's Seventh Framework Programme (FP7/2007-2013) under grant agreement n²28929, acronym NanoDiaRA. The authors thank Katharina Mader for proofreading.

\section{References}

1. Commission Recommendation of 18 October 2011 on the definition of nanomaterial (2011). Official Journal of the European Union. The European Commission,

2. Koodali RT, Klabunde KJ (2012) Nanotechnology: Fundamental Principles and Applications. In: Kent JA (ed) Handbook of Industrial Chemistry and Biotechnology, vol 1. Springer,

3. Boddoes CM, Case CP, Briscoe WH (2015) Understanding nanoparticle cellular entry: A physicochemical perspective. Adv Colloid Interface Sci 218 (C):48-68 
4. Georgieva JV, Hoekstra D, Zuhorn IS (2014) Smuggling Drugs into the Brain: An Overview of Ligands Targeting Transcytosis for Drug Delivery across the Blood-Brain Barrier. Pharmaceutics 6 (4):557-583

5. Wang H, Thorling CA, Liang X, Bridle KR, Grice JE, Zhu Y, Crawford DHG, Xu ZP, Liu X, Roberts MS (2015) Diagnostic imaging and therapeutic application of nanoparticles targeting the liver. Journal of Materials Chemistry B 3 (939-958)

6. Xu C, Sun S (2013) New forms of superparamagnetic nanoparticles for biomedical application. Advanced Drug Delivery Reviews 65 (5):732-743

7. Jin R, Lin B, Li D, Ai H (2014) Superparamagnetic iron oxide nanoparticles for MR imaging and therapy: design considerations and clinical applications. Current Opinion in Pharmacology 18 (October 2014):18-27

8. EMEA (2006) Reflection Paper on Nanotechnology-based Medicinal Products for Human Use. http://www.ema.europa.eu/docs/en GB/document library/Regulatory and procedural guideline/2010 /01/WC500069728.pdf. Accessed December 132015

9. EFSA (2009) The Potential Risks Arising from Nanoscience and Nanotechnologies on Food and Feed Safety. http://www.efsa.europa.eu/de/efsajournal/pub/958. Accessed December 132015

10. FDA (2007) Nanotechnology Task Force Report. Commisioner of Food and Drugs. http://www.fda.gov/ScienceResearch/SpecialTopics/Nanotechnology/UCM2006659.htm.

11. Tolaymat T, El Badawy A, Sequeira R, Genaidy A (2015) An integrated science basedmethodology to assess potential risks and implications of engineered nanomaterials. Journal of Hazardous Materials 298:270-281

12. Reinsborough MT, Sullivan G (2011) The Regulation of Nano-particles under the European Biocidal Products Directive:Challenges for Effective Civil SocietyParticipation. European Journal of Law and Technology 2 (3)

13. Gaspar R (2007) Regulatory issues surrounding nanomedicines: setting the scene for the next generation of nanopharmaceuticals. Nanomedicine (Lond) 2 (2):143-147.

doi:10.2217/17435889.2.2.143

14. Toy R, Peiris PM, Gaghada KB, Karathanasis E (2014) Shaping cancer nanomedicine: the effect of particle shape on the in vivo journey of nanoparticles. Nanomedicine : nanotechnology, biology, and medicine 9 (1):121-134

15. Science and Technology Policy: Nanotechnology. (2012) OECD. http://www.oecd.org/sti/nano/. Accessed November 292014

16. EC (2011) High-Level Expert Group on Key Enabling Technologies - Final Report. European Commission. http://ec.europa.eu/enterprise/sectors/ict/files/kets/hlg_report_final_en.pdf.

17. Preliminary Review of OECD Test Guidelines for their Applicability to Manufactured Nanomaterials (2009). Series on the Safety of Manufactured Nanomaterials, vol 15. Paris 
18. Testing of Chemicals (2013) OECD. http://www.oecd.org/chemicalsafety/testing/. Accessed November 292014

19. ISO/TC 229 Nanotechnologies. (2005) International Organization for Standardization (ISO). http://www.iso.org/iso/iso technical committee?commid=381983. Accessed November 20142014

20. Nanotechnologies - Terminology and definitions for nano-objects - Nanoparticle, nanofibre and nanoplate (2008). vol ISO/TS 27687:2008 International Organization for Standardization (ISO),

21. Guidance for Genotoxicity Tesing and Data Interpretation for Pharmaceuticals Intended for Human Use (2011). vol S2(R1). International Conference on Harmonisation (ICH) - Requirements for Registration of Pharmaceuticals for Human Use,

22. Guidance on the compilation of safety data sheets, version 2.0 (2013). vol ECHA-13-G-11-EN. European Chemicals Agency, Helsinki

23. Das Sicherheitsdatenblatt in der Schweiz basierend auf der 4. Revision der ChemV gültig ab 1.12.2012 (2013). Bundesamt für Gesundheit, Anmeldestelle Chemikalien,

24. Safety data sheet (SDS): Guidelines for synthetic nanomaterials (2012). Federal Department of Economic Affairs DEA - State Secretariat for Economic Affairs SECO, Switzerland

25. Bleeker EAJ, Cassee FR, Geertsma RE, De Jong WH, Heugens EHW, Koers-Jacquemijns M, van De Meent D, Oomen AG, Popma J, Rietveld AG, Wijnhoven SWP (2012) Interpretation and implications of the European Commission Recommendation on the definition of nanomaterial.

26. Rauscher H (2012) The European Commission's recommendation on the definition of nanomaterial makes an impact. Nanotoxicology 7 (7):1195-1197

27. Fulda C, Weber-Bruls D, Werth J (2014) Nano is nano is nano or: nanotechnology - a European legal perspective. Nanotechnology Reviews 3 (4)

28. Hofmann-Amtenbrink M, Hofmann H, Hool A, Roubert F (2014) Nanotechnology in Medicine: European research and its implications. Swiss Medical Weekly 144. doi:10.4414/smw.2014.14044

29. CORDIS (2015) NANODEFINE Periodic Report Summary 1. European Commission. http://cordis.europa.eu/result/rcn/163274_en.html. Accessed May 262015

30. Kurath M, Nentwich M, Fleischer T, Eisenberger I (2014) Cultures and Strategies in the Regulation of Nanotechnology in Germany, Austria, Switzerland and the European Union. NanoEthics 8 (2):121-140

31. Bergkamp L, Michaux G, Herbatschek N (2014) Nanotechnology Regulation in Europe: From REACH and Nano-Registries to Cosmetics, Biocides, and Medical Devices. Nanotechnology Law \& Business $11(1)$

32. REACH Implementation Project on Nanomaterials (RIP-oN) (2009). Substance Identification of Nanomaterials, vol 1. European Commission Joint Research Center - Instiute for Health and Consumer Protection, 
33. REACH Implementation Project on Nanomaterials (RIP-oN) (2011). Specific Advice on Fulfilling Information Requirements for Nanomaterials under REACH, vol 2. European Commission Joint Research Center - Instiute for Health and Consumer Protection,

34. REACH Implementation Project on Nanomaterials (RIP-oN) (2011). Specific Advice on Exposure Assessment and Hazard/Risk Characterisation for Nanomaterials under REACH, vol 3. European Commission Joint Research Center - Instiute for Health and Consumer Protection,

35. Louekari K (2011) Integrated Testing Strategies in REACH. EPAA Workshop IST and their impact on the 3Rs, September 2011, Brussels.

36. NANOREG - A common European approach to the regulatory testing of nanomaterials. (2013) European Commission. http://cordis.europa.eu/project/rcn/107159 en.html. Accessed December 13 2015

37. CORDIS (2015) PROSAFE - Promoting the Implementation of Safe by Design. European Commission. http://cordis.europa.eu/project/rcn/194431 en.html. Accessed May 262015

38. Action plan for synthetic nanomaterials. (2008) Federal Office of Public Health. http://www.bag.admin.ch/nanotechnologie/12167/?lang=en. Accessed November 292014

39. Report of the Federal Council dated 25 April 2012, Action plan for synthetic nanomaterials. (2012) Federal Office of Public Health. http://www.bag.admin.ch/nanotechnologie/12167/?lang=en. Accessed November 292014

40. Guidelines on the Precautionary Matrix for Synthetic Nanomaterials (2008). Federal Office for Public Health and Federal Office for the Environment, Bern

41. Precautionary matrix for synthetic nanomaterials. (2013) Federal Office of Public Health. http://www.bag.admin.ch/nanotechnologie/12171/12174/index.html?lang=de. Accessed November 29 2014

42. EPFL (2013) Directive on the work with engineered nano materials: exposure potential and control measures. vol LEX 1.5.5.

43. Groso A, Petri-Fink A, Magrez A, Riediker M, Meyer T (2010) Management of nanomaterials safety in research environment. Particle and Fibre Toxicology 7 (40)

44. Warheit DB, Borm PJ, Hennes C, Lademann J (2007) Testing strategies to establish the safety of nanomaterials: conclusions of an ECETOC workshop. Inhalation toxicology 19 (8):631-643.

doi:10.1080/08958370701353080

45. Stone V, Nowack B, Baun A, van den Brink N, Kammer F, Dusinska M, Handy R, Hankin S, Hassellov M, Joner E, Fernandes TF (2010) Nanomaterials for environmental studies: classification, reference material issues, and strategies for physico-chemical characterisation. The Science of the total environment 408 (7):1745-1754. doi:10.1016/j.scitotenv.2009.10.035

46. Krug H (2011) Quality Handbook - Standard Procedures for Nanoparticle Testing. http://www.nanopartikel.info/files/methodik/NANOMMUNE-Quality-Handbook-SOPs.pdf. 
47. Aitken RJ, Hankin SM, Tran CL, Donaldson K, Stone V, Cumpson P, Johnstone J, Chaudhry Q, Cash S (2007) REFNANO: Reference materials for engineered nanoparticle toxicology and metrology. Research Project Final Report. defra,

48. DaNa (2009) Methodology of selection, acquisition and evaluation of publications in the Project DaNa. German Ministry of Education and Research.

http://www.nanopartikel.info/files/methodik/DaNa criteria checklist 2015 form.pdf.

49. NANOVALID - Developing Reference Methods for Nanomaterials. (2013).

http://cordis.europa.eu/project/rcn/101043_en.html. Accessed December 132015

50. MARINA - Managing Risks of Nanomaterials. (2013).

http://cordis.europa.eu/project/rcn/93425 en.html. Accessed December 132015

51. Stone V, Pozzi-Mucelli S, Tran L, Aschberger K, Sabella S, Vogel UB, Poland C, Balharry D, Fernandes S, Gottardo S, Hankin S, Hartl M, Hartmann N, Hristozov D, Hund-Rinke K, Johnston H, Marcomini A, Panzer O, Roncato D, Saber AT, Wallin H, Scott-Fordmand JJ (2013) Research Priorisatuin to Deliver and Intelligent Testing Strategy for the Human and Environmental Safety of Nanomaterials. ITS Nano,

52. NANOREG II. (2015). http://cordis.europa.eu/project/rcn/197088 en.html. Accessed December 132015

53. Crist RM, Hall Grossmann J, Patri AK, Stern ST, Dobrovolskaia MA, Adiseshaiah PP, Clogston JD, McNeil SE (2013) Common pitfalls in nanotechnology: lessons learned from NCI's

Nanotechnology Characterization Laboratory. Integrative Biology 5:66-73

54. Joris F, Manshian BB, Peynshaert K, De Smedt SC, Braeckmans K, Soenen SJ (2013) Assessing nanoparticle toxicity in cell-based assays: influence of cell culture parameters and optimized models for bridging the in vitro-in vivo gap. Chemical Society reviews 42 (21):8339-8359.

doi:10.1039/c3cs60145e

55. Warheit DB, Hoke RA, Finlay C, Donner EM, Reed KL, Sayes CM (2007) Development of a base set of toxicity tests using ultrafine $\mathrm{TiO} 2$ particles as a component of nanoparticle risk management. Toxicology letters 171 (3):99-110. doi:10.1016/j.toxlet.2007.04.008

56. Nanotechnology Characterization Laboratory. (2014) National Cancer Institute. http://ncl.cancer.gov. Accessed November 292014

57. Knowledge Base Nanomaterials. (2013). http://nanopartikel.info. Accessed November 292014

58. Terms and definitions used in connection with reference materials (1992). vol ISO Guide 30:1992. International Organization for Standardization (ISO),

59. Nanoscaled Reference Materials. (2009) Federal Institute for Materials Research and Testing BAM. http://www.nano-refmat.bam.de/en/. Accessed November 20142014

60. Kroll A, Pillukat MH, Hahn D, Schnekenburger J (2012) Interference of engineered nanoparticles with in vitro toxicity assays. Arch Toxicol 86 (7):1123-1136. doi:10.1007/s00204-012-0837-z 
61. Nimesh S (2011) Potential implications of nanoparticle characterization on in vitro and in vivo gene delivery. Therapeutic Delivery 3 (11):1347-1356

62. Donaldson K, Murphy FA, Duffin R, Poland CA (2010) Asbestos, carbon nanotubes and the pleural mesothelium: a review of the hypothesis regarding the role of long fibre retention in the parietal pleura, inflammation and mesothelioma. Particle and Fibre Toxicology 7 (5)

63. NIA (2015) US and Canada develop Classification Scheme for Nanomaterials. http://www.nanotechia.org/node/18580. Accessed December 132015

64. Hofmann-Amtenbrink M (2010) NanoDiaRA internal Deliverable D5.6: Guidelines for improving and monitoring research exchange/ethical issues

65. Hool A, Beuzelin Ollivier M-G, Roubert F (2013) Managing Operating Procedures in Distributed Collaborative Projects. Journal of Physics: Conference Series 429

66. Thomas J, Harden A (2008) Methods for the thematic synthesis of qualitative research in systematic reviews. BMC Medical Research Methodology 8 (1):45

67. Hool A, Beuzelin Ollivier M-G, Roubert F (2013) Managing Operating Procedures in Distributed Collaborative Projects. Journal of Physics: Conference Series 429 (012071)

68. NNI (2015) Glossary. National Nanotechnology Institute. http://www.nano.gov/aboutnni/glossary. Accessed December 182015

69. BAG (2011) Gloassary Nanotechnology. Federal Office of Public Health FOPH.

http://www.bag.admin.ch/nanotechnologie/glossar/index.html?lang=en. Accessed December 182015

70. Stefaniak AB, Hackley VA, Roebben G, Ehara K, Hankin S, Postek MT, Lynch I, Fu W-E, Linsiniger TPJ, Thünemann AF (2013) Nanoscale reference materials for environmental, health and safety measurements: needs, gaps and opportunities. Nanotoxicology 7 (8)

71. Hirsch C, Roesslein M, Krug HF, Wick P (2011) Nanomaterial cell interactions: are current in vitro tests reliable? Nanomedicine : nanotechnology, biology, and medicine 6 (5):837-847

72. NANOREG (2014) Factsheets of NANOREG Output. NANOREG - A common European approach to the regulatory testing of nanomaterials. http://nanoreg.eu/index.php/media-anddownloads/factsheets-of-nanoreg-output.html. Accessed December 182015

73. Strehl C, Gaber T, Maurizi L, Hahne M, Rauch R, Hoff P, Häupl T, Hofmann-Amtenbrink M, Poole AR, Hofmann H, Buttgereit F (2015) Effects of PVA coated nanoparticles on human immune cells. International Journal of Nanomedicine 10:3429-3445

74. CCMX (2015) CCMX Winter School 2016. http://www.ccmx.ch/news/newssingle/article/269/31/. Accessed December 132015 